\section{ㄷ REPORTS}

ISSN 1600-5368

\section{Crystal structure of tetraaquabis(8- chloro-9,10-dioxo-9,10-dihydroanthra- cene-1-carboxylato- $\kappa \mathrm{O}^{1}$ ) cobalt(II) dihydrate}

\author{
Wen-Juan Cai, Bo Liu, Feng-Yi Liu and Jun-Feng Kou* \\ College of Chemistry and Chemical Engineering, Yunnan Normal University, \\ Kunming 650500, People's Republic of China. *Correspondence e-mail: \\ kjf416@163.com
}

Received 16 September 2014; accepted 20 September 2014

Edited by T. J. Prior, University of Hull, England

In the title complex, $\left[\mathrm{Co}\left(\mathrm{C}_{15} \mathrm{H}_{6} \mathrm{ClO}_{4}\right)_{2}\left(\mathrm{H}_{2} \mathrm{O}\right)_{4}\right] \cdot 2 \mathrm{H}_{2} \mathrm{O}$, the $\mathrm{Co}^{\mathrm{II}}$ ion is bound by two carboxylate $\mathrm{O}$ atoms of two 5-chloro-9,10anthraquinone-1-carboxylate anions and four water $\mathrm{O}$ atoms in a trans conformation, forming an irregular octahedral coordination geometry. This arrangement is stabilized by intramolecular $\mathrm{O}-\mathrm{H} \cdots \mathrm{O}$ hydrogen bonds between water and carboxylate. Further $\mathrm{O}-\mathrm{H} \cdots \mathrm{O}$ hydrogen bonds between coordinating and non-coordinating water and carboxylate produce layers of molecules that extend parallel to (001). The organic ligands project above and below the plane. Those ligands of adjacent planes are interdigitated and there are $\pi-\pi$ interactions between them with centroid-centroid distances of 3.552 (2) and 3.767 (2) § that generate a three-dimensional supramolecular structure.

Keywords: crystal structure; cobalt; antitumor; hydrogen bond.

CCDC reference: 1025297

\section{Related literature}

For the synthesis of the title complex, see: George et al. (2006). The major advantage of metal-based over organic-based drugs is the ability to vary coordination number, geometry and redox states, and metals can also change the pharmacological properties of organic-based drugs by forming coordination complexes with them, see: Hambley (2007). Anthraquinones are highly effective chemotherapeutic agents with a wide spectrum of antitumor activity, see: Unverferth et al. (1983); Kantrowitz \& Bristow (1984); Stuart et al. (1984); Arcamone (1987). For related compounds, see: Bruijnincx \& Sadler (2008); Gruber et al. (2010); Neufeind et al. (2011).<smiles>O=C(OC(O)(O)OC(O)(O)OC(=O)c1cccc2c1C(=O)c1c(Cl)cccc1C2=O)c1cccc2c1C(=O)c1c(Cl)cccc1C2=O</smiles>

\section{Experimental}

\subsection{Crystal data}

$\left[\mathrm{Co}\left(\mathrm{C}_{15} \mathrm{H}_{6} \mathrm{ClO}_{4}\right)_{2}\left(\mathrm{H}_{2} \mathrm{O}\right)_{4}\right] \cdot 2 \mathrm{H}_{2} \mathrm{O}$

$M_{r}=738.32$

Triclinic, $P \overline{1}$

$a=6.8655$ (14) §

$b=8.1623(16) \AA$

$c=14.285(3) \AA$

$\alpha=73.97(3)^{\circ}$

$\beta=88.86(3)^{\circ}$

\subsection{Data collection}

Rigaku MM007-HF CCD (Saturn 724+) diffractometer

Absorption correction: multi-scan ( ABSCOR; Higashi, 1995)

$T_{\min }=0.837, T_{\max }=0.870$

7246 measured reflections

$$
\begin{aligned}
& \gamma=73.35(3)^{\circ} \\
& V=735.6(3) \AA^{3} \\
& Z=1 \\
& \text { Mo } K \alpha \text { radiation } \\
& \mu=0.84 \mathrm{~mm}^{-1} \\
& T=293 \mathrm{~K} \\
& 0.22 \times 0.19 \times 0.17 \mathrm{~mm}
\end{aligned}
$$
2171 reflections with $I>2 \sigma(I)$ $R_{\text {int }}=0.041$

2 standard reflections every 150 reflections intensity decay: none

\subsection{Refinement}

$R\left[F^{2}>2 \sigma\left(F^{2}\right)\right]=0.052$

$w R\left(F^{2}\right)=0.179$

$S=1.12$

3329 reflections

235 parameters

9 restraints

$\mathrm{H}$ atoms treated by a mixture of

\begin{tabular}{|c|c|c|c|c|}
\hline$D-\mathrm{H} \cdots A$ & $D-\mathrm{H}$ & $\mathrm{H} \cdots A$ & $D \cdots A$ & $D-\mathrm{H} \cdots A$ \\
\hline $\mathrm{O} 7-\mathrm{H} 7 B \cdots \mathrm{O} 3$ & $0.80(4)$ & $2.37(5)$ & $3.121(5)$ & $157(8)$ \\
\hline $\mathrm{O} 7-\mathrm{H} 7 A \cdots \mathrm{O} 4^{\mathrm{i}}$ & $0.82(3)$ & $2.24(4)$ & $3.049(4)$ & $169(8)$ \\
\hline $\mathrm{O} 6-\mathrm{H} 6 B \cdots \mathrm{O} 4$ & $0.82(3)$ & $1.92(3)$ & $2.717(4)$ & $164(5)$ \\
\hline $\mathrm{O} 6-\mathrm{H} 6 A \cdots \mathrm{O} 4^{\mathrm{ii}}$ & $0.82(3)$ & $2.17(4)$ & $2.916(4)$ & $152(6)$ \\
\hline $\mathrm{O} 5-\mathrm{H} 5 B \cdots \mathrm{O} 7^{\mathrm{iii}}$ & $0.78(3)$ & $2.08(4)$ & $2.821(4)$ & $159(5)$ \\
\hline $\mathrm{O} 5-\mathrm{H} 5 A \cdots \mathrm{O} 2$ & $0.81(3)$ & $2.22(4)$ & $2.932(4)$ & $147(5)$ \\
\hline
\end{tabular}
independent and constrained refinement

$\Delta \rho_{\max }=0.59 \mathrm{e}^{-3}$

$\Delta \rho_{\min }=-0.64$ e $\AA^{-3}$

Table 1

Hydrogen-bond geometry $\left(\AA{ }^{\circ}\right)$.

Symmetry codes: (i) $x+1, y, z$; (ii) $-x,-y,-z+1$; (iii) $x, y-1, z$. 
Data collection: CrystalStructure (Rigaku/MSC, 2006); cell refinement: CrystalStructure; data reduction: CrystalStructure; program(s) used to solve structure: SHELXS97 (Sheldrick, 2008); program(s) used to refine structure: SHELXL97 (Sheldrick, 2008); molecular graphics: DIAMOND (Brandenburg, 1999); software used to prepare material for publication: SHELXTL (Sheldrick, 2008).

\section{Acknowledgements}

This work was supported by Natural Science Foundation of Yunnan Provice (grant No. 2009CD048), the Applied Basic Research Projects of Yunnan Provine (grant No. 2014fz042) and the Scientific Research Fund of Yunnan Education (grant No. 2013Y431)

Supporting information for this paper is available from the IUCr electronic archives (Reference: PJ2015).

\section{References}

Arcamone, F. (1987). Cancer Treat. Rev. 25, 2721-2729.

Brandenburg, K. (1999). DIAMOND. Crystal Impact GbR, Bonn, Germany. Bruijnincx, P. C. A. \& Sadler, P. J. (2008). Curr. Opin. Chem. Biol. 12, 197-206.

George, T. A., Hammud, H. H. \& Isber, S. (2006). Polyhedron, 25, 2721-2729.

Gruber, T., Helas, S. F., Seichter, W. \& Weber, E. (2010). Struct. Chem. 21, 1079-1083.

Hambley, T. W. (2007). Science, 318, 1392-1393.

Higashi, T. (1995). ABSCOR. Rigaku Corporation, Tokyo, Japan.

Kantrowitz, N. E. \& Bristow, M. R. (1984). Prog. Cardiovasc. Dis. 27, 195-200.

Neufeind, S., Hülsken, N., Neudörfl, J.-M., Schlörer, N. \& Schmalz, H.-G. (2011). Chem. Eur. J. 17, 2633-2641.

Rigaku/MSC. (2006). CrystalStructure. Rigaku/MSC, The Woodlands, Texas, USA.

Sheldrick, G. M. (2008). Acta Cryst. A64, 112-122.

Stuart, H. A., Peason, M., Smith, I. E. \& Olsen, E. G. J. (1984). Lancet, 2, 219 220.

Unverferth, D. V., Unverferth, B. J., Balcerzak, S. P., Bashore, T. A. \& Neidhart, J. A. (1983). Cancer Treat. Rep. 67, 343-350. 


\section{supporting information}

Acta Cryst. (2014). E70, m357-m358 [doi:10.1107/S1600536814020972]

\section{Crystal structure of tetraaquabis(8-chloro-9,10-dioxo-9,10-dihydro- anthracene-1-carboxylato- $\left.\kappa \mathrm{O}^{1}\right)$ cobalt(II) dihydrate}

\section{Wen-Juan Cai, Bo Liu, Feng-Yi Liu and Jun-Feng Kou}

\section{S1. Comment}

The major advantage of metal-based over organic-based drugs is the ability to vary coordination number, geometry, and redox states and metals can also change the pharmacological properties of organic-based drugs by forming coordination complexes with them. (Hambley et al. 2007) Medicinal inorganic chemistry, covering applications of metals in therapeutics and diagnostics, is a field of increasing prominence (Bruijnincx et al. 2008) after the discovery and successful clinical applications of the Pt-based anticancer drug cisplatin. Anthraquinones are highly effective chemotherapeutic agents with a wide spectrum of antitumor activity. (Unverferth et al. 1983; Kantrowitz et al. 1984; Stuart et al. 1984; Arcamone et al. 1987;). Herein we report the synthesis and structure of the title cobalt(II) anthraquione complex.

The structure of the title complex is shown in Fig. 1, Fig. 2 and hydrogen-bond geometry is given in Table 1. The complex crystallizes in the triclinic space group $P \overline{1}$ and the asymmetric unit consists of one crystallographically independent co(II) cation, one 5-cyclo-9,10-anthraquinone-1-carboxylate anion, two coordination water molecules and one free water molecule. As shown in Fig.1, the $\mathrm{Co}(\mathrm{II})$ ion is bound by two carboxylate $\mathrm{O}$ atom (O3,O3A), four water molecules forming an irregular coordination geometry. Strong hydrogen bonds involving an aqua ligand (as a donor) and carboxy $\mathrm{O}$ atoms (as an acceptor) may further stabilize the three-dimensional structure (O5 $\cdots \mathrm{O} 2=2.932$ (4) $\AA$, $\mathrm{O} 5 \cdots \mathrm{O} 7 \# 1=2.821$ (4) $\AA, \mathrm{O} 6 \cdots \mathrm{O} 4 \# 2=2.916$ (4) $\AA, \mathrm{O} 6 \cdots \mathrm{O} 4=2.717$ (4) $\AA$, symmetry codes: $\# 1 x, y-1, z \# 2-x,-y,-z+1)$. The interstitial water molecules are attached via hydrogen bonding to carboxylate $\mathrm{O}$ atoms $(\mathrm{O} 7 \cdots \mathrm{O} 4 \# 3=3.049$ (4) $\AA$,

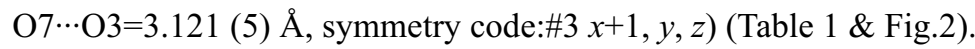

\section{S2. Experimental}

An aqueous solution $(2 \mathrm{ml})$ of cobalt(II) chloride hexahydrate $(0.1 \mathrm{mmol}, 23.7 \mathrm{mg})$ was mixed with a methanolic solution $(2 \mathrm{~mL})$ of 5-cyclo-9,10-anthraquinone-1-carboxylate $(0.1 \mathrm{mmol}, 28.6 \mathrm{mg})$ in presence of two drops of aqueous sodium hydroxide $(0.1 \mathrm{M})$. The resulting mixture was allowed to evaporate for one week to yield red crystals, suitable for X-ray work. Yield: 75\% (based on the 5-cyclo-9,10-anthraquinone-1-carboxylate)

\section{S3. Refinement}

$\mathrm{H}$ atoms attached to carbons were geometrically fixed and allowed to ride on the corresponding non- $\mathrm{H}$ atom with $\mathrm{C}-\mathrm{H}=$ $0.96 \AA$, and $U_{\text {iso }}(\mathrm{H})=1.2 U_{\text {eq }}(\mathrm{C})$ for other $\mathrm{H}$ atoms. For the water molecules, all $\mathrm{O}-\mathrm{H}$ distances were constrained to be equal within a standard deviation of $0.03 \AA$. Similar $\mathrm{H}^{\cdots} \cdot \mathrm{H}$ distance restraints were applied to restrain the bond angle, but with a larger standard deviation. $\mathrm{H}$ atoms of bound water were refined with a single isotropic displacement parameter. Similarly, those of free water were refined with a single, different, $\mathrm{U}_{\text {iso. }}$. 


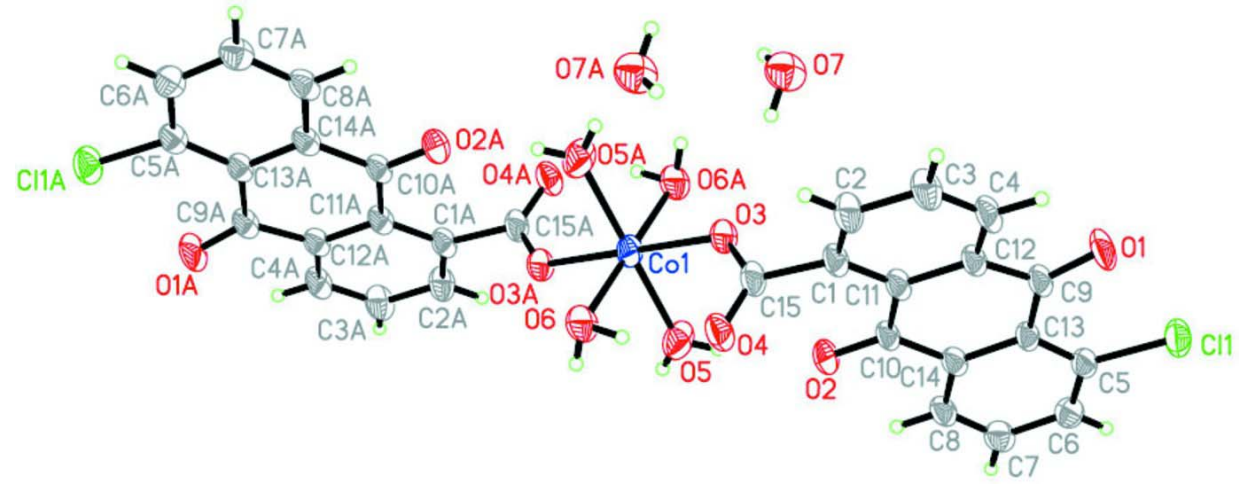

\section{Figure 1}

The molecular structure of the title compound, with atom labels and $30 \%$ probability displacement ellipsoids. Symmetry equivalent atoms labelled with an $\mathrm{A}(\mathrm{eg} \mathrm{O} 1 \mathrm{~A})$ are generated by the symmetry operator $1-x,-y, 1-z$.

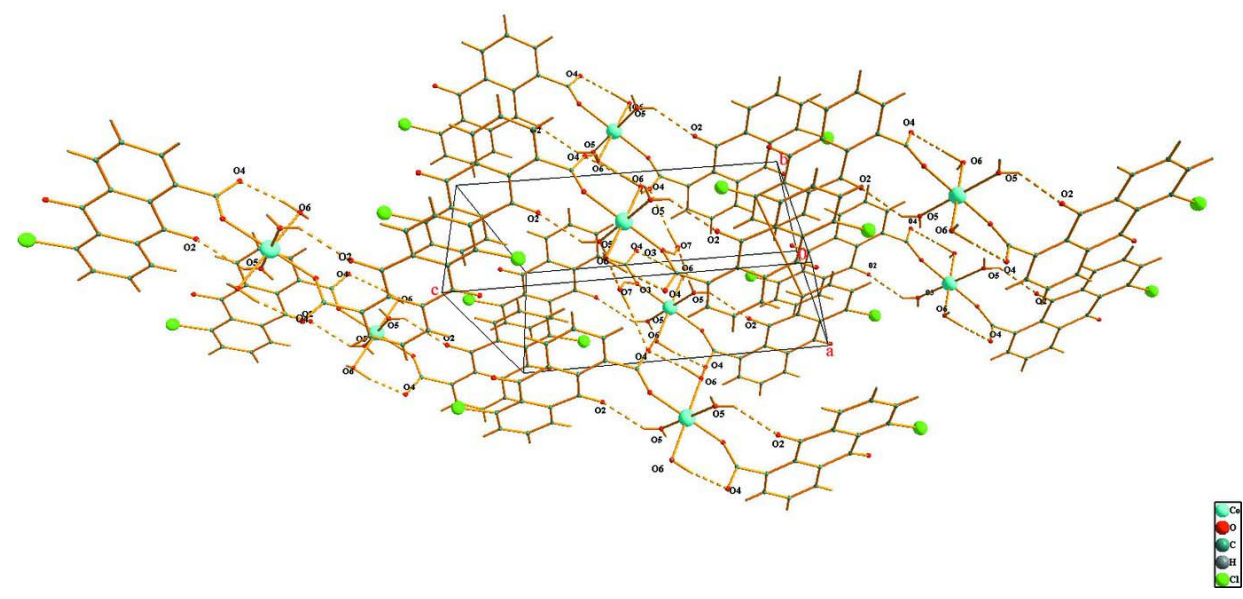

\section{Figure 2}

A view of the crystal packing. Hydrogen bonds are shown as brown dashed lines.

\section{Tetraaquabis(8-chloro-9,10-dioxo-9,10-dihydroanthracene-1-carboxylato- $\left.\kappa O^{1}\right)$ cobalt(II) dihydrate}

\section{Crystal data}

$\left[\mathrm{Co}\left(\mathrm{C}_{15} \mathrm{H}_{6} \mathrm{ClO}_{4}\right)_{2}\left(\mathrm{H}_{2} \mathrm{O}\right)_{4}\right] \cdot 2 \mathrm{H}_{2} \mathrm{O}$

$M_{r}=738.32$

Triclinic, $P \overline{1}$

Hall symbol: -P 1

$a=6.8655$ (14) $\AA$

$b=8.1623(16) \AA$

$c=14.285(3) \AA$

$\alpha=73.97(3)^{\circ}$

$\beta=88.86(3)^{\circ}$

$\gamma=73.35(3)^{\circ}$

$V=735.6(3) \AA^{3}$

\section{Data collection}

Rigaku MM007-HF CCD (Saturn 724+)

diffractometer

Radiation source: rotating anode
$Z=1$

$F(000)=377$

$D_{\mathrm{x}}=1.667 \mathrm{Mg} \mathrm{m}^{-3}$

Mo $K \alpha$ radiation, $\lambda=0.71073 \AA$

Cell parameters from 25 reflections

$\theta=3.1-25.0^{\circ}$

$\mu=0.84 \mathrm{~mm}^{-1}$

$T=293 \mathrm{~K}$

Block, red

$0.22 \times 0.19 \times 0.17 \mathrm{~mm}$

Confocal monochromator $\omega$ scans at fixed $\chi=45^{\circ}$ 
Absorption correction: multi-scan

(ABSCOR; Higashi, 1995)

$T_{\min }=0.837, T_{\max }=0.870$

7246 measured reflections

3329 independent reflections

2171 reflections with $I>2 \sigma(I)$

\section{Refinement}

Refinement on $F^{2}$

Least-squares matrix: full

$R\left[F^{2}>2 \sigma\left(F^{2}\right)\right]=0.052$

$w R\left(F^{2}\right)=0.179$

$S=1.12$

3329 reflections

235 parameters

9 restraints

Primary atom site location: structure-invariant

direct methods

$$
\begin{aligned}
& R_{\text {int }}=0.041 \\
& \theta_{\max }=27.5^{\circ}, \theta_{\min }=3.1^{\circ} \\
& h=-7 \rightarrow 8 \\
& k=-10 \rightarrow 10 \\
& l=-18 \rightarrow 18
\end{aligned}
$$

Secondary atom site location: difference Fourier map

Hydrogen site location: inferred from neighbouring sites

$\mathrm{H}$ atoms treated by a mixture of independent and constrained refinement

$w=1 /\left[\sigma^{2}\left(F_{\mathrm{o}}^{2}\right)+(0.0859 P)^{2}+0.2785 P\right]$ where $P=\left(F_{\mathrm{o}}^{2}+2 F_{\mathrm{c}}^{2}\right) / 3$

$(\Delta / \sigma)_{\max }<0.001$

$\Delta \rho_{\max }=0.59 \mathrm{e} \AA^{-3}$

$\Delta \rho_{\min }=-0.64$ e $\AA^{-3}$

\section{Special details}

Geometry. All e.s.d.'s (except the e.s.d. in the dihedral angle between two 1.s. planes) are estimated using the full covariance matrix. The cell e.s.d.'s are taken into account individually in the estimation of e.s.d.'s in distances, angles and torsion angles; correlations between e.s.d.'s in cell parameters are only used when they are defined by crystal symmetry. An approximate (isotropic) treatment of cell e.s.d.'s is used for estimating e.s.d.'s involving 1.s. planes.

Refinement. Refinement of $F^{2}$ against ALL reflections. The weighted $R$-factor $w R$ and goodness of fit $S$ are based on $F^{2}$, conventional $R$-factors $R$ are based on $F$, with $F$ set to zero for negative $F^{2}$. The threshold expression of $F^{2}>\sigma\left(F^{2}\right)$ is used only for calculating $R$-factors(gt) etc. and is not relevant to the choice of reflections for refinement. $R$-factors based on $F^{2}$ are statistically about twice as large as those based on $F$, and $R$ - factors based on ALL data will be even larger.

Fractional atomic coordinates and isotropic or equivalent isotropic displacement parameters $\left(\AA^{2}\right)$

\begin{tabular}{lllll}
\hline & $x$ & $y$ & $z$ & $U_{\text {iso }} / U_{\text {eq }}$ \\
\hline Co1 & 0.5000 & 0.0000 & 0.5000 & $0.0521(3)$ \\
O1 & $0.2123(5)$ & $0.3055(4)$ & $1.0202(2)$ & $0.0734(8)$ \\
O2 & $0.2651(4)$ & $-0.0771(3)$ & $0.76542(19)$ & $0.0618(7)$ \\
O3 & $0.3637(4)$ & $0.1703(3)$ & $0.58318(18)$ & $0.0565(6)$ \\
O4 & $0.0299(4)$ & $0.1985(4)$ & $0.5694(2)$ & $0.0631(7)$ \\
O5 & $0.4564(6)$ & $-0.2191(4)$ & $0.6077(2)$ & $0.0798(10)$ \\
H5A & $0.431(8)$ & $-0.222(7)$ & $0.663(3)$ & $0.101(9)^{*}$ \\
H5B & $0.469(8)$ & $-0.317(5)$ & $0.607(4)$ & $0.101(9)^{*}$ \\
O6 & $0.2118(5)$ & $0.0420(4)$ & $0.4326(2)$ & $0.0664(8)$ \\
H6A & $0.181(8)$ & $-0.050(6)$ & $0.442(4)$ & $0.101(9)^{*}$ \\
H6B & $0.141(7)$ & $0.079(7)$ & $0.474(3)$ & $0.101(9)^{*}$ \\
O7 & $0.6143(6)$ & $0.4206(5)$ & $0.6095(3)$ & $0.0832(9)$ \\
H7A & $0.724(7)$ & $0.371(10)$ & $0.591(6)$ & $0.16(2)^{*}$ \\
H7B & $0.527(8)$ & $0.385(10)$ & $0.594(6)$ & $0.16(2)^{*}$ \\
C1 & $0.1499(5)$ & $0.3019(5)$ & $0.6896(3)$ & $0.0497(8)$ \\
C2 & $0.0846(6)$ & $0.4864(5)$ & $0.6614(3)$ & $0.0624(10)$ \\
H2 & 0.0601 & 0.5482 & 0.5955 & $0.075^{*}$ \\
C3 & $0.0552(7)$ & $0.5804(5)$ & $0.7312(3)$ & $0.0666(11)$
\end{tabular}




$\begin{array}{lllll}\text { H3 } & 0.0103 & 0.7043 & 0.7116 & 0.080^{*} \\ \text { C4 } & 0.0924(6) & 0.4908(5) & 0.8284(3) & 0.0590(10) \\ \text { H4 } & 0.0715 & 0.5541 & 0.8746 & 0.071^{*} \\ \text { C5 } & 0.2727(5) & -0.0750(5) & 1.0984(3) & 0.0506(8) \\ \text { C6 } & 0.3092(5) & -0.2598(5) & 1.1281(3) & 0.0583(9) \\ \text { H6 } & 0.3266 & -0.3212 & 1.1942 & 0.070^{*} \\ \text { C7 } & 0.3193(6) & -0.3514(5) & 1.0589(3) & 0.0619(10) \\ \text { H7 } & 0.3390 & -0.4738 & 1.0783 & 0.074^{*} \\ \text { C8 } & 0.3001(6) & -0.2605(5) & 0.9614(3) & 0.0562(9) \\ \text { H8 } & 0.3107 & -0.3230 & 0.9152 & 0.067^{*} \\ \text { C9 } & 0.2065(5) & 0.2169(5) & 0.9648(3) & 0.0508(8) \\ \text { C10 } & 0.2436(5) & 0.0117(5) & 0.8235(3) & 0.0466(8) \\ \text { C11 } & 0.1858(5) & 0.2093(5) & 0.7891(3) & 0.0476(8) \\ \text { C12 } & 0.1613(5) & 0.3057(5) & 0.8584(3) & 0.0482(8) \\ \text { C13 } & 0.2495(5) & 0.0193(5) & 1.0001(2) & 0.0459(8) \\ \text { C14 } & 0.2651(5) & -0.0773(5) & 0.9307(3) & 0.0477(8) \\ \text { C15 } & 0.1838(6) & 0.2122(5) & 0.6087(3) & 0.0525(8) \\ \text { C11 } & 0.25829(17) & 0.02230(15) & 1.19317(7) & 0.0696(3)\end{array}$

Atomic displacement parameters $\left(\AA^{2}\right)$

\begin{tabular}{lllllll}
\hline & $U^{11}$ & $U^{22}$ & $U^{33}$ & $U^{12}$ & $U^{13}$ & $U^{23}$ \\
\hline Co1 & $0.0629(5)$ & $0.0479(4)$ & $0.0531(4)$ & $-0.0121(3)$ & $0.0195(3)$ & $-0.0316(3)$ \\
O1 & $0.106(2)$ & $0.0652(17)$ & $0.0646(17)$ & $-0.0219(16)$ & $0.0088(16)$ & $-0.0472(14)$ \\
O2 & $0.0817(18)$ & $0.0561(14)$ & $0.0632(16)$ & $-0.0195(13)$ & $0.0199(13)$ & $-0.0434(12)$ \\
O3 & $0.0627(15)$ & $0.0581(15)$ & $0.0598(15)$ & $-0.0146(12)$ & $0.0214(12)$ & $-0.0389(12)$ \\
O4 & $0.0655(16)$ & $0.0728(18)$ & $0.0649(16)$ & $-0.0171(14)$ & $0.0081(13)$ & $-0.0453(14)$ \\
O5 & $0.121(3)$ & $0.0548(16)$ & $0.0705(19)$ & $-0.0255(17)$ & $0.0424(19)$ & $-0.0311(15)$ \\
O6 & $0.0744(19)$ & $0.0636(17)$ & $0.0722(19)$ & $-0.0160(14)$ & $0.0169(14)$ & $-0.0419(15)$ \\
O7 & $0.083(2)$ & $0.065(2)$ & $0.104(3)$ & $-0.0127(17)$ & $0.012(2)$ & $-0.0370(18)$ \\
C1 & $0.0487(18)$ & $0.0543(19)$ & $0.055(2)$ & $-0.0088(15)$ & $0.0092(15)$ & $-0.0359(16)$ \\
C2 & $0.076(3)$ & $0.054(2)$ & $0.058(2)$ & $-0.0064(19)$ & $0.0075(19)$ & $-0.0311(18)$ \\
C3 & $0.082(3)$ & $0.050(2)$ & $0.073(3)$ & $-0.0062(19)$ & $0.009(2)$ & $-0.0377(19)$ \\
C4 & $0.067(2)$ & $0.056(2)$ & $0.066(2)$ & $-0.0103(18)$ & $0.0129(19)$ & $-0.0445(19)$ \\
C5 & $0.0410(17)$ & $0.065(2)$ & $0.056(2)$ & $-0.0157(16)$ & $0.0121(15)$ & $-0.0355(17)$ \\
C6 & $0.054(2)$ & $0.063(2)$ & $0.062(2)$ & $-0.0151(18)$ & $0.0108(17)$ & $-0.0269(18)$ \\
C7 & $0.061(2)$ & $0.055(2)$ & $0.075(3)$ & $-0.0146(18)$ & $0.0123(19)$ & $-0.0302(19)$ \\
C8 & $0.055(2)$ & $0.058(2)$ & $0.070(2)$ & $-0.0168(17)$ & $0.0136(18)$ & $-0.0410(19)$ \\
C9 & $0.0468(18)$ & $0.058(2)$ & $0.061(2)$ & $-0.0128(15)$ & $0.0140(16)$ & $-0.0421(17)$ \\
C10 & $0.0423(17)$ & $0.0548(19)$ & $0.057(2)$ & $-0.0143(15)$ & $0.0128(15)$ & $-0.0385(16)$ \\
C11 & $0.0428(17)$ & $0.0524(18)$ & $0.060(2)$ & $-0.0111(15)$ & $0.0124(15)$ & $-0.0387(16)$ \\
C12 & $0.0446(17)$ & $0.0546(19)$ & $0.058(2)$ & $-0.0133(15)$ & $0.0136(15)$ & $-0.0384(16)$ \\
C13 & $0.0394(16)$ & $0.0551(19)$ & $0.0528(19)$ & $-0.0117(14)$ & $0.0112(14)$ & $-0.0332(16)$ \\
C14 & $0.0404(16)$ & $0.0548(19)$ & $0.059(2)$ & $-0.0126(15)$ & $0.0102(15)$ & $-0.0346(16)$ \\
C15 & $0.065(2)$ & $0.0494(19)$ & $0.0489(19)$ & $-0.0108(17)$ & $0.0078(17)$ & $-0.0300(15)$ \\
C11 & $0.0773(7)$ & $0.0832(7)$ & $0.0594(6)$ & $-0.0203(6)$ & $0.0136(5)$ & $-0.0419(5)$ \\
& & & & & & \\
\hline
\end{tabular}


Geometric parameters $\left(\AA,{ }^{\circ}\right)$

\begin{tabular}{|c|c|c|c|}
\hline $\mathrm{Co} 1-\mathrm{O}^{\mathrm{i}}$ & $2.083(2)$ & $\mathrm{C} 2-\mathrm{H} 2$ & 0.9300 \\
\hline $\mathrm{Co} 1-\mathrm{O} 3$ & $2.083(2)$ & $\mathrm{C} 3-\mathrm{C} 4$ & $1.369(6)$ \\
\hline $\mathrm{Co} 1-\mathrm{O}^{\mathrm{i}}$ & $2.104(3)$ & $\mathrm{C} 3-\mathrm{H} 3$ & 0.9300 \\
\hline $\mathrm{Co} 1-\mathrm{O} 5$ & $2.104(3)$ & $\mathrm{C} 4-\mathrm{C} 12$ & $1.390(5)$ \\
\hline $\mathrm{Co} 1-\mathrm{O}^{\mathrm{i}}$ & $2.113(3)$ & $\mathrm{C} 4-\mathrm{H} 4$ & 0.9300 \\
\hline $\mathrm{Co} 1-\mathrm{O} 6$ & $2.113(3)$ & $\mathrm{C} 5-\mathrm{C} 13$ & $1.390(5)$ \\
\hline $\mathrm{O} 1-\mathrm{C} 9$ & $1.219(4)$ & $\mathrm{C} 5-\mathrm{C} 6$ & $1.397(5)$ \\
\hline $\mathrm{O} 2-\mathrm{C} 10$ & $1.225(4)$ & $\mathrm{C} 5-\mathrm{Cl1}$ & $1.737(3)$ \\
\hline $\mathrm{O} 3-\mathrm{C} 15$ & $1.259(4)$ & $\mathrm{C} 6-\mathrm{C} 7$ & $1.385(5)$ \\
\hline $\mathrm{O} 4-\mathrm{C} 15$ & $1.253(4)$ & C6- $\mathrm{H} 6$ & 0.9300 \\
\hline $\mathrm{O} 5-\mathrm{H} 5 \mathrm{~A}$ & $0.81(3)$ & $\mathrm{C} 7-\mathrm{C} 8$ & $1.373(6)$ \\
\hline $\mathrm{O} 5-\mathrm{H} 5 \mathrm{~B}$ & $0.78(3)$ & $\mathrm{C} 7-\mathrm{H} 7$ & 0.9300 \\
\hline O6-H6A & $0.82(3)$ & $\mathrm{C} 8-\mathrm{C} 14$ & $1.387(5)$ \\
\hline O6- $-\mathrm{H} 6 \mathrm{~B}$ & $0.82(3)$ & $\mathrm{C} 8-\mathrm{H} 8$ & 0.9300 \\
\hline $\mathrm{O} 7-\mathrm{H} 7 \mathrm{~A}$ & $0.82(3)$ & $\mathrm{C} 9-\mathrm{C} 12$ & $1.487(5)$ \\
\hline $\mathrm{O} 7-\mathrm{H} 7 \mathrm{~B}$ & $0.80(4)$ & $\mathrm{C} 9-\mathrm{C} 13$ & $1.492(5)$ \\
\hline $\mathrm{C} 1-\mathrm{C} 2$ & $1.385(5)$ & $\mathrm{C} 10-\mathrm{C} 11$ & $1.485(5)$ \\
\hline $\mathrm{C} 1-\mathrm{C} 11$ & $1.402(5)$ & $\mathrm{C} 10-\mathrm{C} 14$ & $1.493(5)$ \\
\hline $\mathrm{C} 1-\mathrm{C} 15$ & $1.511(4)$ & $\mathrm{C} 11-\mathrm{C} 12$ & $1.405(4)$ \\
\hline $\mathrm{C} 2-\mathrm{C} 3$ & $1.395(5)$ & $\mathrm{C} 13-\mathrm{C} 14$ & $1.412(4)$ \\
\hline $\mathrm{O} 3{ }^{\mathrm{i}}-\mathrm{Co} 1-\mathrm{O} 3$ & $180.00(10)$ & $\mathrm{C} 12-\mathrm{C} 4-\mathrm{H} 4$ & 119.8 \\
\hline $\mathrm{O} 3^{\mathrm{i}}-\mathrm{Co} 1-\mathrm{O}^{\mathrm{i}}$ & $90.64(11)$ & $\mathrm{C} 13-\mathrm{C} 5-\mathrm{C} 6$ & $121.2(3)$ \\
\hline $\mathrm{O} 3-\mathrm{Co} 1-\mathrm{O} 5^{\mathrm{i}}$ & $89.36(11)$ & $\mathrm{C} 13-\mathrm{C} 5-\mathrm{Cl1}$ & $124.1(3)$ \\
\hline $\mathrm{O} 3^{\mathrm{i}}-\mathrm{Co} 1-\mathrm{O} 5$ & $89.36(11)$ & $\mathrm{C} 6-\mathrm{C} 5-\mathrm{Cl} 1$ & $114.7(3)$ \\
\hline $\mathrm{O} 3-\mathrm{Co} 1-\mathrm{O} 5$ & $90.64(11)$ & $\mathrm{C} 7-\mathrm{C} 6-\mathrm{C} 5$ & $119.8(4)$ \\
\hline $\mathrm{O} 5-\mathrm{Co} 1-\mathrm{O} 5$ & 180.0 & $\mathrm{C} 7-\mathrm{C} 6-\mathrm{H} 6$ & 120.1 \\
\hline $\mathrm{O} 3^{\mathrm{i}}-\mathrm{Co} 1-\mathrm{O}^{\mathrm{i}}$ & $89.78(11)$ & $\mathrm{C} 5-\mathrm{C} 6-\mathrm{H} 6$ & 120.1 \\
\hline $\mathrm{O} 3-\mathrm{Co} 1-\mathrm{O}^{\mathrm{i}}$ & $90.22(11)$ & $\mathrm{C} 8-\mathrm{C} 7-\mathrm{C} 6$ & $119.7(4)$ \\
\hline $\mathrm{O} 5^{\mathrm{i}}-\mathrm{Co} 1-\mathrm{O}^{\mathrm{i}}$ & $89.38(15)$ & $\mathrm{C} 8-\mathrm{C} 7-\mathrm{H} 7$ & 120.1 \\
\hline $\mathrm{O} 5-\mathrm{Co} 1-\mathrm{O}^{\mathrm{i}}$ & $90.62(15)$ & $\mathrm{C} 6-\mathrm{C} 7-\mathrm{H} 7$ & 120.1 \\
\hline $\mathrm{O} 3^{\mathrm{i}}-\mathrm{Co} 1-\mathrm{O} 6$ & $90.22(11)$ & $\mathrm{C} 7-\mathrm{C} 8-\mathrm{C} 14$ & $121.2(3)$ \\
\hline $\mathrm{O} 3-\mathrm{Co} 1-\mathrm{O} 6$ & $89.78(11)$ & $\mathrm{C} 7-\mathrm{C} 8-\mathrm{H} 8$ & 119.4 \\
\hline $\mathrm{O} 5^{\mathrm{i}}-\mathrm{Co} 1-\mathrm{O} 6$ & $90.62(15)$ & $\mathrm{C} 14-\mathrm{C} 8-\mathrm{H} 8$ & 119.4 \\
\hline $\mathrm{O} 5-\mathrm{Co} 1-\mathrm{O} 6$ & $89.38(15)$ & $\mathrm{O} 1-\mathrm{C} 9-\mathrm{C} 12$ & $119.7(3)$ \\
\hline $\mathrm{O}^{\mathrm{i}}-\mathrm{Co} 1-\mathrm{O} 6$ & 180.0 & $\mathrm{O} 1-\mathrm{C} 9-\mathrm{C} 13$ & $121.8(3)$ \\
\hline $\mathrm{C} 15-\mathrm{O} 3-\mathrm{Co} 1$ & $130.3(2)$ & $\mathrm{C} 12-\mathrm{C} 9-\mathrm{C} 13$ & $118.4(3)$ \\
\hline $\mathrm{Co} 1-\mathrm{O} 5-\mathrm{H} 5 \mathrm{~A}$ & $126(4)$ & $\mathrm{O} 2-\mathrm{C} 10-\mathrm{C} 11$ & $120.9(3)$ \\
\hline $\mathrm{Co} 1-\mathrm{O} 5-\mathrm{H} 5 \mathrm{~B}$ & $131(4)$ & $\mathrm{O} 2-\mathrm{C} 10-\mathrm{C} 14$ & $120.3(3)$ \\
\hline $\mathrm{H} 5 \mathrm{~A}-\mathrm{O} 5-\mathrm{H} 5 \mathrm{~B}$ & $103(4)$ & $\mathrm{C} 11-\mathrm{C} 10-\mathrm{C} 14$ & $118.7(3)$ \\
\hline $\mathrm{Co} 1-\mathrm{O} 6-\mathrm{H} 6 \mathrm{~A}$ & $112(4)$ & $\mathrm{C} 1-\mathrm{C} 11-\mathrm{C} 12$ & $119.4(3)$ \\
\hline $\mathrm{Co} 1-\mathrm{O} 6-\mathrm{H} 6 \mathrm{~B}$ & $98(4)$ & $\mathrm{C} 1-\mathrm{C} 11-\mathrm{C} 10$ & $121.7(3)$ \\
\hline $\mathrm{H} 6 \mathrm{~A}-\mathrm{O} 6-\mathrm{H} 6 \mathrm{~B}$ & $96(4)$ & $\mathrm{C} 12-\mathrm{C} 11-\mathrm{C} 10$ & $118.9(3)$ \\
\hline $\mathrm{H} 7 \mathrm{~A}-\mathrm{O} 7-\mathrm{H} 7 \mathrm{~B}$ & $110(5)$ & $\mathrm{C} 4-\mathrm{C} 12-\mathrm{C} 11$ & $120.0(3)$ \\
\hline $\mathrm{C} 2-\mathrm{C} 1-\mathrm{C} 11$ & $119.5(3)$ & $\mathrm{C} 4-\mathrm{C} 12-\mathrm{C} 9$ & $117.6(3)$ \\
\hline $\mathrm{C} 2-\mathrm{C} 1-\mathrm{C} 15$ & 116.7 & $\mathrm{C} 11-\mathrm{C} 12-\mathrm{C} 9$ & $122.4(3)$ \\
\hline
\end{tabular}




\begin{tabular}{|c|c|c|c|}
\hline $\mathrm{C} 11-\mathrm{C} 1-\mathrm{C} 15$ & $123.8(3)$ & $\mathrm{C} 5-\mathrm{C} 13-\mathrm{C} 14$ & $118.0(3)$ \\
\hline $\mathrm{C} 1-\mathrm{C} 2-\mathrm{C} 3$ & $120.6(4)$ & $\mathrm{C} 5-\mathrm{C} 13-\mathrm{C} 9$ & $123.2(3)$ \\
\hline $\mathrm{C} 1-\mathrm{C} 2-\mathrm{H} 2$ & 119.7 & $\mathrm{C} 14-\mathrm{C} 13-\mathrm{C} 9$ & $118.8(3)$ \\
\hline $\mathrm{C} 3-\mathrm{C} 2-\mathrm{H} 2$ & 119.7 & $\mathrm{C} 8-\mathrm{C} 14-\mathrm{C} 13$ & $120.1(3)$ \\
\hline $\mathrm{C} 4-\mathrm{C} 3-\mathrm{C} 2$ & $120.2(4)$ & $\mathrm{C} 8-\mathrm{C} 14-\mathrm{C} 10$ & $117.9(3)$ \\
\hline $\mathrm{C} 4-\mathrm{C} 3-\mathrm{H} 3$ & 119.9 & $\mathrm{C} 13-\mathrm{C} 14-\mathrm{C} 10$ & $122.1(3)$ \\
\hline $\mathrm{C} 2-\mathrm{C} 3-\mathrm{H} 3$ & 119.9 & $\mathrm{O} 4-\mathrm{C} 15-\mathrm{O} 3$ & $126.6(3)$ \\
\hline $\mathrm{C} 3-\mathrm{C} 4-\mathrm{C} 12$ & $120.3(3)$ & $\mathrm{O} 4-\mathrm{C} 15-\mathrm{C} 1$ & $117.3(3)$ \\
\hline $\mathrm{C} 3-\mathrm{C} 4-\mathrm{H} 4$ & 119.8 & $\mathrm{O} 3-\mathrm{C} 15-\mathrm{C} 1$ & $115.9(3)$ \\
\hline $\mathrm{O} 3{ }^{\mathrm{i}}-\mathrm{Co} 1-\mathrm{O} 3-\mathrm{C} 15$ & $-8(100)$ & $\mathrm{C} 13-\mathrm{C} 9-\mathrm{C} 12-\mathrm{C} 4$ & $170.7(3)$ \\
\hline $\mathrm{O} 5-\mathrm{Co} 1-\mathrm{O} 3-\mathrm{C} 15$ & $115.4(3)$ & $\mathrm{O} 1-\mathrm{C} 9-\mathrm{C} 12-\mathrm{C} 11$ & $169.1(3)$ \\
\hline $\mathrm{O} 5-\mathrm{Co} 1-\mathrm{O} 3-\mathrm{C} 15$ & $-64.6(3)$ & $\mathrm{C} 13-\mathrm{C} 9-\mathrm{C} 12-\mathrm{C} 11$ & $-9.3(5)$ \\
\hline $\mathrm{O} 6-\mathrm{Co} 1-\mathrm{O} 3-\mathrm{C} 15$ & $-155.2(3)$ & $\mathrm{C} 6-\mathrm{C} 5-\mathrm{C} 13-\mathrm{C} 14$ & $-0.3(5)$ \\
\hline $\mathrm{O} 6-\mathrm{Co} 1-\mathrm{O} 3-\mathrm{C} 15$ & $24.8(3)$ & $\mathrm{C} 11-\mathrm{C} 5-\mathrm{C} 13-\mathrm{C} 14$ & $180.0(2)$ \\
\hline $\mathrm{C} 11-\mathrm{C} 1-\mathrm{C} 2-\mathrm{C} 3$ & $0.3(6)$ & $\mathrm{C} 6-\mathrm{C} 5-\mathrm{C} 13-\mathrm{C} 9$ & $178.8(3)$ \\
\hline $\mathrm{C} 15-\mathrm{C} 1-\mathrm{C} 2-\mathrm{C} 3$ & $-178.1(4)$ & $\mathrm{Cl1}-\mathrm{C} 5-\mathrm{C} 13-\mathrm{C} 9$ & $-0.9(5)$ \\
\hline $\mathrm{C} 1-\mathrm{C} 2-\mathrm{C} 3-\mathrm{C} 4$ & $0.5(7)$ & $\mathrm{O} 1-\mathrm{C} 9-\mathrm{C} 13-\mathrm{C} 5$ & $8.6(5)$ \\
\hline $\mathrm{C} 2-\mathrm{C} 3-\mathrm{C} 4-\mathrm{C} 12$ & $0.5(6)$ & $\mathrm{C} 12-\mathrm{C} 9-\mathrm{C} 13-\mathrm{C} 5$ & $-173.1(3)$ \\
\hline $\mathrm{C} 13-\mathrm{C} 5-\mathrm{C} 6-\mathrm{C} 7$ & $-1.1(6)$ & $\mathrm{O} 1-\mathrm{C} 9-\mathrm{C} 13-\mathrm{C} 14$ & $-172.2(3)$ \\
\hline $\mathrm{C} 11-\mathrm{C} 5-\mathrm{C} 6-\mathrm{C} 7$ & $178.6(3)$ & $\mathrm{C} 12-\mathrm{C} 9-\mathrm{C} 13-\mathrm{C} 14$ & $6.1(5)$ \\
\hline $\mathrm{C} 5-\mathrm{C} 6-\mathrm{C} 7-\mathrm{C} 8$ & $2.1(6)$ & $\mathrm{C} 7-\mathrm{C} 8-\mathrm{C} 14-\mathrm{C} 13$ & $0.3(6)$ \\
\hline $\mathrm{C} 6-\mathrm{C} 7-\mathrm{C} 8-\mathrm{C} 14$ & $-1.7(6)$ & $\mathrm{C} 7-\mathrm{C} 8-\mathrm{C} 14-\mathrm{C} 10$ & $-179.3(3)$ \\
\hline $\mathrm{C} 2-\mathrm{C} 1-\mathrm{C} 11-\mathrm{C} 12$ & $-2.1(5)$ & $\mathrm{C} 5-\mathrm{C} 13-\mathrm{C} 14-\mathrm{C} 8$ & $0.8(5)$ \\
\hline $\mathrm{C} 15-\mathrm{C} 1-\mathrm{C} 11-\mathrm{C} 12$ & $176.1(3)$ & $\mathrm{C} 9-\mathrm{C} 13-\mathrm{C} 14-\mathrm{C} 8$ & $-178.5(3)$ \\
\hline $\mathrm{C} 2-\mathrm{C} 1-\mathrm{C} 11-\mathrm{C} 10$ & $176.2(3)$ & $\mathrm{C} 5-\mathrm{C} 13-\mathrm{C} 14-\mathrm{C} 10$ & $-179.7(3)$ \\
\hline $\mathrm{C} 15-\mathrm{C} 1-\mathrm{C} 11-\mathrm{C} 10$ & $-5.6(5)$ & $\mathrm{C} 9-\mathrm{C} 13-\mathrm{C} 14-\mathrm{C} 10$ & $1.1(5)$ \\
\hline $\mathrm{O} 2-\mathrm{C} 10-\mathrm{C} 11-\mathrm{C} 1$ & $1.2(5)$ & $\mathrm{O} 2-\mathrm{C} 10-\mathrm{C} 14-\mathrm{C} 8$ & $-3.0(5)$ \\
\hline $\mathrm{C} 14-\mathrm{C} 10-\mathrm{C} 11-\mathrm{C} 1$ & $-175.8(3)$ & $\mathrm{C} 11-\mathrm{C} 10-\mathrm{C} 14-\mathrm{C} 8$ & $174.0(3)$ \\
\hline $\mathrm{O} 2-\mathrm{C} 10-\mathrm{C} 11-\mathrm{C} 12$ & $179.5(3)$ & $\mathrm{O} 2-\mathrm{C} 10-\mathrm{C} 14-\mathrm{C} 13$ & $177.4(3)$ \\
\hline $\mathrm{C} 14-\mathrm{C} 10-\mathrm{C} 11-\mathrm{C} 12$ & $2.5(5)$ & $\mathrm{C} 11-\mathrm{C} 10-\mathrm{C} 14-\mathrm{C} 13$ & $-5.6(5)$ \\
\hline $\mathrm{C} 3-\mathrm{C} 4-\mathrm{C} 12-\mathrm{C} 11$ & $-2.4(6)$ & $\mathrm{Co} 1-\mathrm{O} 3-\mathrm{C} 15-\mathrm{O} 4$ & $-20.4(6)$ \\
\hline $\mathrm{C} 3-\mathrm{C} 4-\mathrm{C} 12-\mathrm{C} 9$ & $177.6(4)$ & $\mathrm{Co} 1-\mathrm{O} 3-\mathrm{C} 15-\mathrm{C} 1$ & $165.0(2)$ \\
\hline $\mathrm{C} 1-\mathrm{C} 11-\mathrm{C} 12-\mathrm{C} 4$ & $3.2(5)$ & $\mathrm{C} 2-\mathrm{C} 1-\mathrm{C} 15-\mathrm{O} 4$ & $-80.0(5)$ \\
\hline $\mathrm{C} 10-\mathrm{C} 11-\mathrm{C} 12-\mathrm{C} 4$ & $-175.2(3)$ & $\mathrm{C} 11-\mathrm{C} 1-\mathrm{C} 15-\mathrm{O} 4$ & $101.7(4)$ \\
\hline $\mathrm{C} 1-\mathrm{C} 11-\mathrm{C} 12-\mathrm{C} 9$ & $-176.8(3)$ & $\mathrm{C} 2-\mathrm{C} 1-\mathrm{C} 15-\mathrm{O} 3$ & $95.1(4)$ \\
\hline $\mathrm{C} 10-\mathrm{C} 11-\mathrm{C} 12-\mathrm{C} 9$ & $4.8(5)$ & $\mathrm{C} 11-\mathrm{C} 1-\mathrm{C} 15-\mathrm{O} 3$ & $-83.2(4)$ \\
\hline $\mathrm{O} 1-\mathrm{C} 9-\mathrm{C} 12-\mathrm{C} 4$ & $-11.0(5)$ & & \\
\hline
\end{tabular}

Symmetry code: (i) $-x+1,-y,-z+1$.

Hydrogen-bond geometry $\left(\AA,{ }^{\circ}\right)$

\begin{tabular}{lllll}
\hline$D-\mathrm{H} \cdots A$ & $D-\mathrm{H}$ & $\mathrm{H} \cdots A$ & $D \cdots A$ & $D-\mathrm{H} \cdots A$ \\
\hline $\mathrm{O} 7-\mathrm{H} 7 B \cdots \mathrm{O} 3$ & $0.80(4)$ & $2.37(5)$ & $3.121(5)$ & $157(8)$ \\
$\mathrm{O} 7-\mathrm{H} 7 A \cdots \mathrm{O} 4{ }^{\mathrm{ii}}$ & $0.82(3)$ & $2.24(4)$ & $3.049(4)$ & $169(8)$ \\
$\mathrm{O} 6-\mathrm{H} 6 B \cdots \mathrm{O} 4$ & $0.82(3)$ & $1.92(3)$ & $2.717(4)$ & $164(5)$ \\
$\mathrm{O} 6-\mathrm{H} 6 A \cdots \mathrm{O} 4{ }^{\mathrm{iii}}$ & $0.82(3)$ & $2.17(4)$ & $2.916(4)$ & $152(6)$
\end{tabular}


supporting information

$\mathrm{O} 5-\mathrm{H} 5 B \cdots \mathrm{O} 7^{\mathrm{iv}}$

$0.78(3)$

$2.08(4)$

$2.821(4)$

$2.22(4)$

$2.932(4)$

$159(5)$

$\mathrm{O} 5-\mathrm{H} 5 A \cdots \mathrm{O} 2$

$0.81(3)$

4)

Symmetry codes: (ii) $x+1, y, z$; (iii) $-x,-y,-z+1$; (iv) $x, y-1, z$. 